\title{
Acoustic phonons and a central mode in the protonic conductor $\mathrm{K}_{3} \mathrm{H}\left(\mathrm{SeO}_{4}\right)_{2}$
}

\author{
Fumihito Shikanai, ${ }^{*}$ Shinya Tsukada, Jun Kano, and Seiji Kojima ${ }^{\dagger}$ \\ Graduate School of Pure and Applied Sciences, University of Tsukuba, Tsukuba, Ibaraki 305-8573, Japan \\ (Received 1 September 2009; revised manuscript received 8 October 2009; published 12 January 2010)
}

\begin{abstract}
The dynamical properties of protonic conductor $\mathrm{K}_{3} \mathrm{H}\left(\mathrm{SeO}_{4}\right)_{2}$ were examined by Brillouin scattering. Both longitudinal-acoustic modes $\left(c_{33}\right.$ and $\left.c_{11}\right)$ and a transversal mode $\left(c_{44}\right)$ monotonously depend on temperature, and no soft mode was observed. A central mode with a width about $6 \mathrm{GHz}$ was observed above phase-transition temperature, $T_{\mathrm{C}}$. The central mode has a symmetry $E_{\mathrm{g}}$ corresponding to the rotational mode of $\mathrm{SeO}_{4}$ tetrahedra. The $E_{\mathrm{g}}$ mode is compatible with a zone-boundary soft mode, $L_{3}$, which accounts for the symmetrical change from $R \overline{3} m$ to $C 2 / c$. The intensity of the central mode declines in the vicinity of $T_{\mathrm{C}}$. This shows that chaotic rotational motion changes into a zone-boundary soft mode with decreasing temperature.
\end{abstract}

DOI: 10.1103/PhysRevB.81.012301

PACS number(s): 66.30.H-, 77.84.Fa, 78.35.+c

Development of solid electrolytes for fuel cells that work at a medium temperature range is desired. Oxide ceramics used in a solid oxide fuel cells (SOFCs) show self-catalysis at operating temperatures greater than $600{ }^{\circ} \mathrm{C}$. Because the electrical energy is transformed from the chemical reaction energy of oxygen and hydrogen, high-temperature operation is preferable. However, at high temperatures, problems such as the deterioration of electrodes occur. On the other hand, a polymer electrolyte membrane (PEM) shows marked high electrical conductivity of more than $10^{-2} \mathrm{~S} / \mathrm{cm}$. However, PEMs must be operated below $100{ }^{\circ} \mathrm{C}$ because the protons are transferred by water. Some hydrogen-bonded anhydrous materials represented as $M_{\mathrm{HXO}}$ and $M_{3} \mathrm{H}\left(X_{4}\right)_{2} \quad(M$ $=\mathrm{K}, \mathrm{Rb}, \mathrm{Cs}, \mathrm{NH}_{4}$ and $X=\mathrm{S}, \mathrm{Se}$ ) may attempt to solve this problem. Most of them undergo phase transitions above $150{ }^{\circ} \mathrm{C}$ and show high electrical conductivity of about $10^{-3}$ to $10^{-2} \mathrm{~S} / \mathrm{cm}$ without decomposition even in humid atmosphere despite their water solubility. ${ }^{1}$ Application of their proton conduction mechanism to a new solid electrolyte is expected to facilitate development of a next-generation fuel cell; therefore, the clarification of the mechanism is required urgently.

$\mathrm{K}_{3} \mathrm{H}\left(\mathrm{SeO}_{4}\right)_{2}$ (TKHSe) is one of the $M_{3} \mathrm{H}\left(X_{4}\right)_{2}$-type compounds that has been investigated in terms of the phasetransition mechanism for years. ${ }^{2-7}$ The material undergoes a first-order phase transition at $390 \mathrm{~K}\left(T_{\mathrm{C}}\right)$; it shows a remarkably high electrical conductivity of about $10^{-3} \mathrm{~S} / \mathrm{cm}$, above $T_{\mathrm{C}}$ (phase I) and is ferroelastic below $T_{\mathrm{C}}$ (phase II). ${ }^{8}$ The crystal belongs to the trigonal system with the space group $R \overline{3} m$ in phase I and to monoclinic $C 2 / c$ in phase II. An isolated $\mathrm{SeO}_{4}-\mathrm{H}-\mathrm{SeO}_{4}$ dimer characterizes the crystal structure in phase II, ${ }^{9,10}$ and no interdimer hydrogen bond exists. The dimers construct a layered structure parallel to the monoclinic $b_{\mathrm{m}} c_{\mathrm{m}}$-plane [see Figs. 3(d) and 3(e)] and form a deformed hexagon by the pseudo $\overline{3}$ axis, which is perpendicular to the plane. The hydrogen bonds form a herringbone pattern in the layer in the direction $c_{\mathrm{m}}+b_{\mathrm{m}}$ or $c_{\mathrm{m}}-b_{\mathrm{m}}$; no pattern exists in the direction $-c_{\mathrm{m}}$. In phase $\mathrm{I}$, the distances from a $\mathrm{SeO}_{4}$ tetrahedron to the nearest three tetrahedra are equivalent; and a tetrahedron is linked to one of them with a probability 1/3. ${ }^{11,12}$ Many proton transfer models that show disconnection and reconstruction of hydrogen bond accompanied by the deformation ${ }^{13-16}$ or rotation ${ }^{17}$ of tetrahedra have been proposed. However, the evidence for the dynamical properties of tetrahedra have not been reported in detail.

In addition, the crystal structure in phase II is the superlattice structure of that in phase I. The lattice interval in the direction of trigonal $b_{t}$ in phase II modulates to twice that in phase I. This shows that the ferroelastic phase transition is an improper type occurring at the zone-boundary $L$-point $0 \mathrm{k} / 2$ $0 .{ }^{18}$ Recently, we performed inelastic neutron-scattering (INS) (Ref. 11) and quasielastic neutron-scattering (QENS) (Ref. 19) experiments on TKHSe. From INS, a broad spectrum was observed around $0 \mathrm{meV}$ at $L$ point 0.520 in phase I. The spectrum increases and narrows with decreasing temperature and accounts for the soft mode by an overdamped rotational tetrahedra mode. The full width at half maximum (FWHM) is 70(6) $\mu \mathrm{eV}$ at $435 \mathrm{~K}$. On the other hand, from QENS, a spectrum due to the large incoherent-scattering length of proton was observed. The FWHM of the spectrum depends on both temperature and scattering vector, $Q$; the maximum value estimated from deconvolution of instrumental function is $20(12) \mu \mathrm{eV}$ at $413 \mathrm{~K}$ and $1.65 \AA^{-1}$. What needs to be emphasized is that both spectra have been observed at extremely low energy, within $100 \mu \mathrm{eV}$ (24.2 $\mathrm{GHz}$ ). This indicates that similar excitation in the low-energy region by protons or tetrahedra also exist at the $\Gamma$ point. Furthermore, the temperature dependence of the acoustic mode in the vicinity of the $\Gamma$ point is important for characterizing the ferroelastic phase transition. Therefore, we performed Brillouin scattering experiment on TKHSe, which has high resolution and signal-to-noise ratio.

The present Brief Report reports the acoustic and central mode (CM) of TKHSe in the low-energy region. We show the temperature dependence of the acoustic mode, which is closely related to the ferroelastic phase transition. In addition, we show details of the $\mathrm{CM}$ found above $T_{\mathrm{C}}$ and discuss its origin based on its symmetry and temperature dependence.

Single crystals of TKHSe were grown at $313 \mathrm{~K}$ from an aqueous solution by slow evaporation method. A transparent singlecrystal $1.3 \times 1.0 \times 0.9 \mathrm{~mm}^{3}$ in size was used as a specimen. The light source was a diode-pumped solid-state laser with a single frequency, $\lambda=532 \mathrm{~nm}$. Light-scattering measurements were performed using a high-contrast 3+3-pass tandem Fabry-Pérot interferometer (JRS Science 


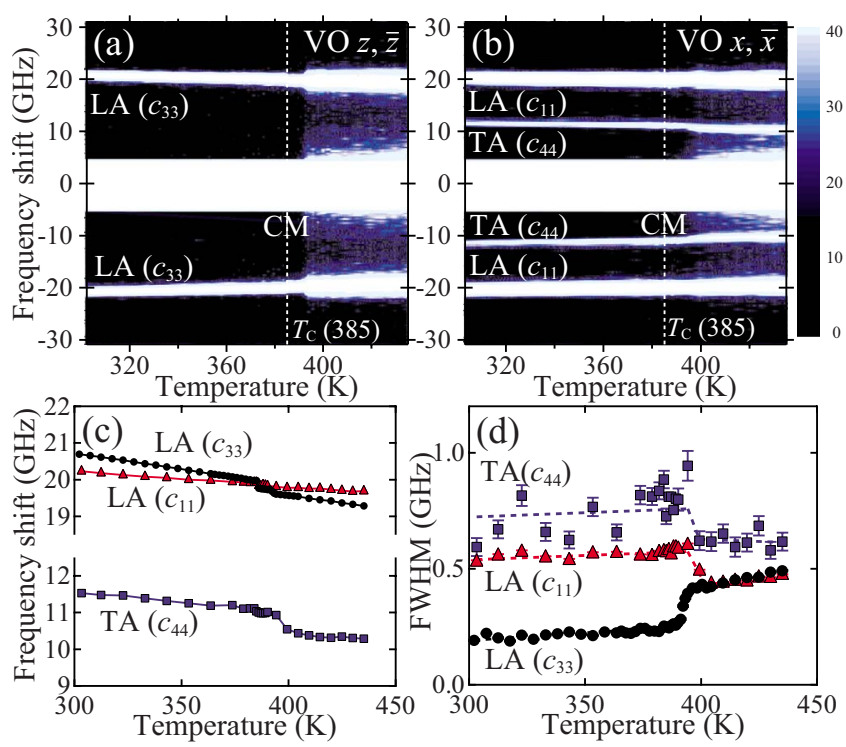

FIG. 1. (Color online) Temperature dependence of inelastic light scattering of $\mathrm{K}_{3} \mathrm{H}\left(\mathrm{SeO}_{4}\right)_{2}$. (a) $\mathrm{VO} z, \bar{z}$. (b) $\mathrm{VO} x, \bar{x}$. (c) Frequency shift of acoustic mode. (d) FWHM of acoustic mode.

Instruments $)^{20}$ and found a $180^{\circ}$ scattering geometry, under polarization conditions without a polarizer [vertical to open (VO)], for the acoustic mode. The data were recorded during cooling at various temperatures from 435 to $300 \mathrm{~K}$. An additional experiment has been performed to measure the $\mathrm{CM}$ above $T_{\mathrm{C}}$. Two other polarization conditions, parallel polarizer [vertical to vertical (VV)] and cross polarizer [vertical to horizontal $(\mathrm{VH})]$, were used to examine the symmetry of the $\mathrm{CM}$ spectrum at $429 \mathrm{~K}$. The phase-transition temperature $T_{\mathrm{C}}$ was $385 \mathrm{~K}$ due to thermal hysteresis during cooling measurement. The lattice axes with no subscript described in the text are those of a trigonal cell (hexagonal setting) in phase I, and $x, y$, and $z$ were defined as $x=a+b=b_{\mathrm{m}}, y=b-a=c_{\mathrm{m}}$, and $z$ $=c=a_{\mathrm{m}}^{*}$, respectively. The relationship between the hexagonal and trigonal settings are $a=a_{t}-b_{t}, b=b_{t}-c_{t}$, and $c=a_{t}$ $+b_{t}+c_{t}$.

Figure 1(a) and 1(b) shows the contour maps of lightscattering intensity versus frequency shift and temperature. A longitudinal-acoustic (LA) mode is observed at the frequency shift $\pm 20.691(1) \mathrm{GHz}$ at $303 \mathrm{~K}$ [Fig. 1(a)]. From Christoffel's equation, $\rho v^{2} \pi_{i}=c_{i j k l} a_{j} \pi_{k} a_{l} \quad(i, j, k, l=1,2,3)$, the LA mode is assigned as a pure longitudinal wave relating to the stiffness $c_{33}$, where $\pi_{i}$ and $a_{i}$ are the polarizing vector and unit scattering vector, respectively. Similarly, a LA and a transversal acoustic (TA) mode were observed at the frequency shift $\pm 20.234(4)$ and $\pm 11.53(2) \mathrm{GHz}$, respectively, at $303 \mathrm{~K}$ [Fig. 1(b)]. Similar calculation shows that the LA mode is the pure longitudinal wave related to $c_{11}$ and the TA mode is a quasitransversal wave $c_{44}+\Delta \simeq c_{44}$, where $\Delta$ $=c_{14}^{2}\left\{4\left(c_{44}-c_{66}\right)\right\}^{-1}$ under the conditions $c_{14} \ll c_{44}-c_{66}$ and $c_{66}=\left(c_{11}-c_{12}\right) / 2$. Hence, the main diagonal components of the stiffness for $R \overline{3} m\left(c_{11}, c_{33}\right.$, and $\left.c_{44}\right)$ were measured under these scattering geometries.

A Voigt function was used to analyze these spectrum. The FWHM of the Gaussian component was fixed as the instrumental function 0.4698(2) GHz. The temperature depen-



FIG. 2. (Color online) The polarization character of CM at 429 K. (a) VV $z(y, y) \bar{z}$. (b) $\mathrm{VH} z(y, x) \bar{z}$. (c) $\operatorname{VV} x(z, z) \bar{x}$. (d) $\mathrm{VH} x(z, y) \bar{x}$.

dence of the frequency shift of each acoustic mode is shown in Fig. 1(c). The frequency shift, $\nu_{\mathrm{B}}$, of $c_{33}$ monotonously increases with decreasing temperature and breaks at $T_{\mathrm{C}}$ with a frequency shift of about $0.2 \mathrm{GHz}$. The other acoustic modes, $c_{11}$ and $c_{44}$ show a similar tendency; however, the steps are at a slightly higher temperature, about $400 \mathrm{~K}$. The velocities of LA and TA at $415 \mathrm{~K}$, obtained using the equation $v=\lambda \nu_{\mathrm{B}}(2 n)^{-1}$, are 3449.3(2) and 3504.3(4) $\mathrm{m} / \mathrm{s}$ for $c_{11}$ and $c_{33}$, respectively, and $1832(2) \mathrm{m} / \mathrm{s}$ for $c_{44}$. Here, the refractivity as that of a typical hydrogen-bonded material, $n$ $=1.5$, was assumed. These values agree with those obtained from dispersion curve by INS, ${ }^{11} 3130(132)$ and $1930(15) \mathrm{m} / \mathrm{s}$ for LA and TA, respectively. The stiffness at $415 \mathrm{~K}$ obtained by substituting these velocities for $c_{i j}=\rho v^{2}$ is $c_{11}=3.361(3)$ $\times 10^{10}, \quad c_{33}=3.748(9) \times 10^{10}$, and $c_{44}=1.024(3) \times 10^{10} \mathrm{~Pa}$, where $\rho=3.052 \mathrm{~g} / \mathrm{cm}$.

Figure 1(d) shows the temperature dependence of the FWHM of the acoustic modes. The FWHM of $c_{33}$ decreases with decreasing temperature and is narrow $(0.17 \mathrm{GHz})$ at $T_{\mathrm{C}}$. Assuming that the reactivity scarcely depends on temperature, the damping constant of $c_{33}$ at $435 \mathrm{~K}$ is 2.76(5) times that at $303 \mathrm{~K}$. The dependence of $c_{11}$ contrasts with that of $c_{33}$. It agrees with that of $c_{33}$ above $T_{\mathrm{C}}$ but broadens below $T_{\mathrm{C}}$. The dependence on $c_{44}$ shows a similar tendency. This broadening of $c_{11}$ and $c_{44}$ is considered to indicate split of the signal due to the domain shift during cooling measurement. The hexagonal $a$ in phase I becomes a nonequivalent axis, $c_{\mathrm{m}}+b_{\mathrm{m}}, c_{\mathrm{m}}-b_{\mathrm{m}}$, or $-c_{\mathrm{m}}$ in phase II, while the hexagonal $c$, common to both domains, appears as monoclinic $a_{\mathrm{m}}^{*}$ in phase II. A warming measurement using a single-domain crystal is needed, although a small contribution will be expected for the phase transition. As mentioned above, the acoustic modes depend monotonously on temperature and no soft mode was observed, although the material shows ferroelasticity in phase II.

In addition to these acoustic modes, as shown in both Figs. 1(a) and 1(b), a spectrum of the CM appears within $\pm 10 \mathrm{GHz}$ above $T_{\mathrm{C}}$. Figure 2 shows the polarization character of the CM at $429 \mathrm{~K}$. The intensity of the CM is modu- 


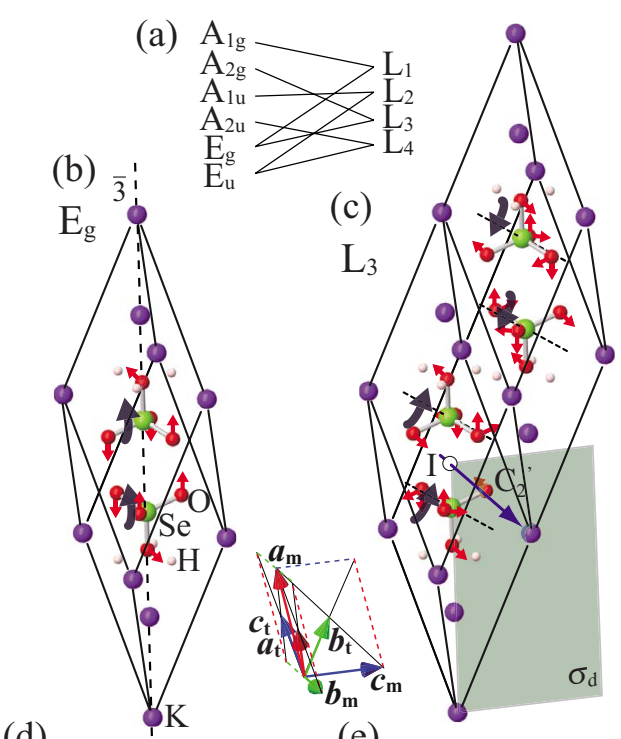

(d)

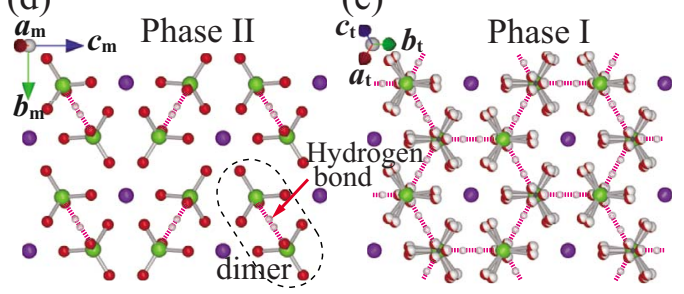

FIG. 3. (Color online) (a) Compatibility conditions between the $\Gamma$ - and $L$-point of $R \overline{3} m$. (b), (c) Atomic displacement patterns of $E_{\mathrm{g}}$ and $L_{3}$ modes. (d), (e) Hydrogen bond arrangements in the layer in phase II and I, respectively. The structures are illustrated using the atomic positions in Ref. 11

lated under each polarization condition, indicating that the $\mathrm{CM}$ is symmetric. The CM spectrum is clearly observed under the polarization conditions in Figs 2(a) and 2(d). In Fig. 2(b), the spectrum significantly decreases, although a small component of CM is remains, which disappears in Fig. 2(c).

There exist two types of Raman-active modes for $R \overline{3} m\left(A_{1 \mathrm{~g}}\right.$ and degenerating $E_{\mathrm{g}}$ ). The Raman tensors are given as follows:

$$
A_{1 \mathrm{~g}}=\left(\begin{array}{ccc}
a & 0 & 0 \\
0 & a & 0 \\
0 & 0 & b
\end{array}\right), \quad E_{\mathrm{g} 1}=\left(\begin{array}{ccc}
c & 0 & 0 \\
0 & -c & d \\
0 & d & 0
\end{array}\right),
$$

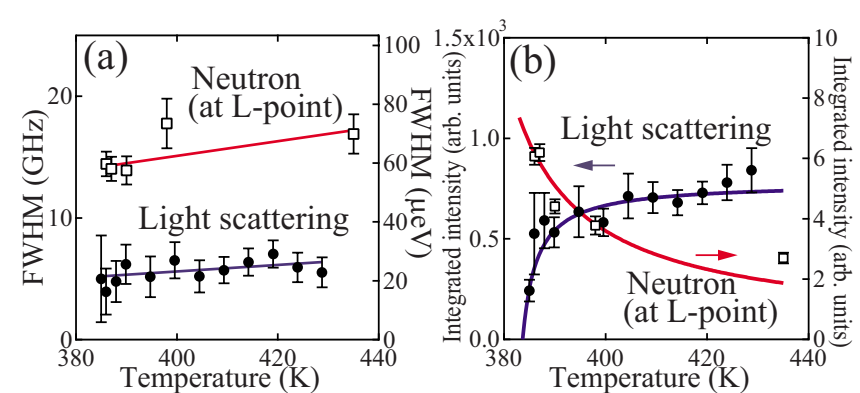

FIG. 4. (Color online) Temperature dependence of CM. (a) FWHM. (b) Integrated intensity. Data denoted by $\square$ s are those at the $L$ point obtained by INS (Ref. 11).

$$
E_{\mathrm{g} 2}=\left(\begin{array}{ccc}
0 & -c & -d \\
-c & 0 & 0 \\
-d & 0 & 0
\end{array}\right)
$$

The quadratic form of the incident and scattering polarizing vectors and matrix of $E_{\mathrm{g} 1}$ gives $-c, 0,0$, and $d$ for the polarization conditions described in Figs. 2(a)-2(d) $(a, 0, b$, and 0 for $A_{1 \mathrm{~g}}$ and $0,-c, 0$, and 0 for $E_{\mathrm{g} 2}$ ). Therefore, the symmetry of the $\mathrm{CM}$ is $E_{\mathrm{g} 1}$, and the small spectrum in Fig. 2(b) originates from the degenerating mode $E_{\mathrm{g} 2}$.

The atomic displacement pattern of $E_{\mathrm{g}}$ mode was obtained by a projection operator at the $\Gamma$-point of $R \overline{3} m$, for arbitrary atomic displacements. The result is shown by red arrows in Fig. 3(b). The main symmetry elements of $R \overline{3} \mathrm{~m}$ are illustrated in Figs. 3(b) and 3(c). The $E_{\mathrm{g}}$ mode is the degenerate rotational mode [Fig. 3(b)]. Two $\mathrm{SeO}_{4}$ tetrahedra in a unit cell rotate in the same phase, shown as black arrows, and incline in the direction of each hydrogen bond in phase II [see Fig. 3(d)]. On the other hand, from our latest INS experiment, a spectrum having a Lorentz functional shape has been observed around $0 \mathrm{meV}$ at the zone-boundary $L$ point and assigned to the $L_{3}$ mode. The definition of $L_{n}$ is listed in the character table (Table I). Freezing of the $L_{3}$ mode accounts for the symmetrical change from $R \overline{3} m$ to maximal subgroup $C 2 / c$. As illustrated in Fig. 3(c), the $L_{3}$ mode also corresponds to the rotational mode of tetrahedra with wavelength $2 b_{t}$. The rotational axes are bisectors of the O-Se-O angles. Figure 3(a) shows the compatibility conditions between the $\Gamma$ and $L$ points. As described in Fig. 3(a), the $E_{\mathrm{g}}$ representation is compatible with the $L_{3}$ representation. Therefore, both the $E_{\mathrm{g}}$ and the $L_{3}$ originate from the same family of modes except for those of wavelength. Further-

\begin{tabular}{|c|c|c|c|c|c|c|c|c|c|}
\hline & $E$ & $C_{2}^{\prime}$ & $I$ & $\sigma_{d}$ & $\left\{E \mid b_{t}\right\}$ & $\left\{C_{2}^{\prime} \mid b_{t}\right\}$ & $\left\{I \mid b_{t}\right\}$ & $\left\{\sigma_{d} \mid b_{t}\right\}$ & \\
\hline$L_{1}$ & 1 & 1 & 1 & 1 & -1 & -1 & -1 & -1 & $\mathrm{C} 2 / \mathrm{m}$ \\
\hline$L_{2}$ & 1 & 1 & -1 & -1 & -1 & -1 & 1 & 1 & $C 2 / c$ \\
\hline$L_{3}$ & 1 & -1 & 1 & -1 & -1 & 1 & -1 & 1 & $C 2 / c$ \\
\hline$L_{4}$ & 1 & -1 & -1 & 1 & -1 & 1 & 1 & -1 & $C 2 / m$ \\
\hline
\end{tabular}

TABLE I. Character table at $L$ point of $R \overline{3} m$ with maximal sub groups of each irreducible representation. 
more, the peak shapes and their position, $0 \mathrm{GHz}$, show that both modes are overdamped.

Figure 4(a) shows the temperature dependence of the FWHM of the CM. The FWHM decreases slightly with temperature from $6.4(1.2) \mathrm{GHz}$ at $429 \mathrm{~K}$ to $5.2(1.9) \mathrm{GHz}$ at 386 $\mathrm{K}$. These values correspond to the lifetime of the CM, $1.5(3) \times 10^{-10}$ and $1.9(7) \times 10^{-10} \mathrm{~s}$ at 429 and $386 \mathrm{~K}$, respectively. The data denoted by open squares in Fig. 4(a) show the FWHM of spectra at the $L$-point obtained by INS. ${ }^{11}$ The FWHM is 60(4) and 70(6) $\mu \mathrm{eV}$ at 386 and $435 \mathrm{~K}$, respectively, and its lifetimes are $7.1(4) \times 10^{-11} \mathrm{~s}$ to $5.9(4)$ $\times 10^{-11} \mathrm{~s}$. Although the FWHM values are about 2.5 times larger than that obtained by light scattering because of the resolution of INS, these lifetimes of about $10^{-10} \mathrm{~s}$ are consistent with each other. They are also consistent with the relaxation time of transfer protons obtained by other experiments such as NMR in $\mathrm{Rb}_{3} \mathrm{H}\left(\mathrm{SO}_{4}\right)_{2},{ }^{21} 9.54 \times 10^{-10} \mathrm{~s}$ at 435 $\mathrm{K}$ (the value was calculated from the activation energy, $E_{\mathrm{a}}$ $=25 \mathrm{KJ}^{-1} \mathrm{~mol}^{-1}$, and the inverse of a frequency factor, $\tau_{0}$ $\left.=9.5 \times 10^{-13} \mathrm{~s}\right)$, and QENS, ${ }^{19} 4.9(3) \times 10^{-11} \mathrm{~s}$ at $433 \mathrm{~K}$. This shows that proton conduction is simultaneous with the motion of the rotational mode.

Figure 4(b) shows the temperature dependence of the integrated intensity of $\mathrm{CM}$ above $T_{\mathrm{C}}$. The intensity of $\mathrm{CM}$ gradually decreases with decreasing temperature and fades out below $400 \mathrm{~K}$. This temperature dependence differs from that of the relaxation mode observed in the representative hydrogen-bonded material $\mathrm{KH}_{2} \mathrm{PO}_{4}$. The fitting curve used in Fig. 4(b) is $A\left\{1-B T /\left(T-T_{0}\right)\right\}$ with three parameters, $A, B$, and $T_{0}$. An extrapolated Curie temperature $T_{0}$ of $381(3) \mathrm{K}$ was obtained. The data denoted by open squares in Fig. 4(b) are those at the $L$ point. ${ }^{11}$ In contrast to the CM, the data increase with decreasing temperature as $C T /\left(T-T_{0}\right)$, that is, both intensities show complementary temperature dependence. This shows that the overdamped rotational mode is chaotic at sufficiently high temperature, and changes into a zone-boundary phonon mode with decreasing temperature.

In this Brief Report, we showed acoustic and optical excitations of a protonic conductor, TKHSe, in a low-energy region thorough light scattering. Although the material shows ferroelasticity in phase II, both the TA and LA modes show no significant anomaly in a wide temperature region including the vicinity of $T_{\mathrm{C}}$. On the other hand, a Lorentzianshaped spectrum with a FWHM of $6 \mathrm{GHz}$ appears around 0 $\mathrm{GHz}$ as the $\mathrm{CM}$ above $T_{\mathrm{C}}$. The $\mathrm{CM}$ was assigned as an overdamped rotational mode of $\mathrm{SeO}_{4}$ tetrahedra which has the symmetry $E_{\mathrm{g}}$. The $E_{\mathrm{g}}$ mode is compatible with a zoneboundary mode, $L_{3}$, which plays a role of a soft mode and causes the improper ferroelastic phase transition. The intensity of CM declines in the vicinity of $T_{\mathrm{C}}$, while it increases at $L$ point. These results reveal the role of each atom. The potassium atom makes a rigid frame, which is evident as the lack of anomaly in the acoustic modes. The rotational motion of tetrahedra contributes largely to the proton conductivity and phase transition. Chaotic rotational motion of $\mathrm{SeO}_{4}$ tetrahedra with a lifetime of about $10^{-10}$ s changes into a zoneboundary mode, making local dimer structures with transfer protons in the same time scale.

\footnotetext{
*Present address: Private, 2-15-11-102 Chikuho, Tsukuba, Ibaraki 300-3253, Japan. fumihito@star.dti2.ne.jp

†kojima@bk.tsukuba.ac.jp

${ }^{1}$ S. M. Haile, D. A. Boysen, C. R. I. Chisholm, and R. B. Merle, Nature (London) 410, 910 (2001).

${ }^{2}$ Y. Noda, H. Kasatani, Y. Watanabe, and H. Terauchi, J. Phys. Soc. Jpn. 61, 905 (1992).

${ }^{3}$ C. Totsuji and T. Matsubara, J. Phys. Soc. Jpn. 63, 2760 (1994).

${ }^{4}$ Y. Suwa, J. Yamaguchi, H. Kageshima, and S. Tsuneyuki, Mater. Sci. Eng., B 79, 31 (2001).

${ }^{5}$ F. Fillaux, A. Lautié, J. Tomkinson, and G. J. Kearly, Chem. Phys. 154, 135 (1991)

${ }^{6}$ Y. Moritomo, Y. Tokura, N. Nagaosa, T. Suzuki, and K. Kumagai, Phys. Rev. Lett. 71, 2833 (1993).

${ }^{7}$ P. Kaung, M. Kasahara, and T. Yagi, J. Phys. Soc. Jpn. 65, 1114 (1996).

${ }^{8}$ J. Hatori, Y. Matsuo, and S. Ikehata, Solid State Commun. 140, 452 (2006)

${ }^{9}$ M. Ichikawa, S. Sato, M. Komukae, and T. Osaka, Acta Crystallogr., Sect. C: Cryst. Struct. Commun. 48, 1569 (1992).

${ }^{10}$ N. Onoda-Yamamuro, O. Yamamuro, T. Matsuo, M. Ichikawa,
}

R. M. Ibbreson, and W. I. F. David, J. Phys.: Condens. Matter 12, 8559 (2000).

${ }^{11}$ F. Shikanai, K. Tomiyasu, N. Aso, S. Ikeda, and T. Kamiyama, Phys. Rev. B 80, 144103 (2009).

${ }^{12}$ F. Shikanai, R. Kiyanagi, M. Yonemura, D. Sulistyanintyas, K. Iwase, T. Wuernisha, K. Mori, T. Ishigaki, S. Ikeda, and T. Kamiyama, Physica B 385-386, 156 (2006).

${ }^{13}$ B. V. Merinov, A. I. Baranov, and L. A. Shuvalov, Sov. Phys. Crystallogr. 35, 200 (1990).

${ }^{14}$ A. Bohn, R. Melzer, R. Sonntag, R. E. Lechner, G. Schuc, and K. Langer, Solid State Ionics 77, 111 (1995).

${ }^{15}$ R. E. Lechner, Solid State Ionics 145, 167 (2001).

${ }^{16}$ N. I. Pavlenko and I. V. Stasyuk, J. Chem. Phys. 114, 4607 (2001).

${ }^{17}$ H. Kamimura, Y. Matsuo, S. Ikehata, T. Ito, M. Komukae, and T. Osaka, Phys. Status Solidi B 241, 61 (2004).

${ }^{18}$ S. Yokota, Y. Makita, and Y. Takagi, J. Phys. Soc. Jpn. 51, 1461 (1982).

${ }^{19}$ F. Shikanai et al., Ferroelectrics 347, 74 (2007).

${ }^{20}$ F. M. Jiang and S. Kojima, Appl. Phys. Lett. 77, 1271 (2000).

${ }^{21}$ K. I. Suzuki and S. Hayashi, Phys. Rev. B 73, 024305 (2006). 